\title{
Luteal Phase Support in In Vitro Fertilization
}

\author{
Elena H. Yanushpolsky, MD ${ }^{1}$ \\ ${ }^{1}$ Department of Obstetrics and Gynecology, Brigham and Women's \\ Hospital, Harvard Medical School, Boston, Massachusetts \\ Semin Reprod Med 2015;33:118-127
}

\begin{abstract}
Address for correspondence Elena H. Yanushpolsky, MD, Department of Obstetrics and Gynecology, Brigham and Women's Hospital, Harvard Medical School, 75 Francis Street, Boston, Massachusetts 02115 (e-mail: eyanushpolsky@partners.org).
\end{abstract}

\begin{abstract}
Keywords

- luteal phase support

- IVF

- progesterone

- hCG

It has been well demonstrated that luteal phase physiology is disrupted in in vitro fertilization (IVF) cycles conducted with either gonadotropin-releasing hormone ( $\mathrm{GnRH}$ ) agonists or antagonists, and that supplementation of the luteal phase with either exogenous progesterone or human chorionic gonadotropin (hCG) is necessary to optimize IVF cycle outcomes. Though both progesterone and hCG supplementation resulted in comparable pregnancy rates, hCG supplementation was associated with increased risk for ovarian hyperstimulation syndrome (OHSS). For that reason progesterone has been used for luteal support by most IVF programs around the world. Vaginal progesterone preparations have been shown definitively to be equally efficacious and better tolerated by patients than intramuscular progesterone injections, but new data on the subcutaneous and oral progesterone are also emerging. New evidence has been accumulating on the benefits of low-dose luteal hCG supplementation in $\mathrm{GnRH}$ antagonist cycles where $\mathrm{GnRH}$ agonists are used for the final maturation trigger. New approaches to luteal phase support as well as new formulations of progesterone have been developed since the last comprehensive review was published in 2011. In this article, we examine current evidence for efficacy, dosing, and timing of progesterone preparations as well as the role of hCG for luteal support in IVF cycles triggered with $\mathrm{GnRH}$ agonists. We also discuss the data on the role of estrogen supplementation in the luteal phase, optimal duration of progesterone support in early pregnancy, and progesterone replacement in frozen embryo transfer cycles and donor egg recipient cycles.
\end{abstract}

Establishment of successful pregnancy in natural ovulatory cycles depends in large part on adequate endometrial development that is induced by sequential and combined exposures to estrogen and progesterone, which are produced by the developing follicle prior to ovulation and by the corpus luteum after ovulation. ${ }^{1}$ Both follicular estrogen production and corpus luteum function are governed by pulsatile $\mathrm{GnRH}$ output from the hypothalamus. ${ }^{1}$ Stimulated in vitro fertilization (IVF) cycles are different from natural cycles in supraphysiologic estrogen levels in the follicular phase and rapid changes in the estrogen and progesterone levels in the luteal phase after follicle aspiration. Removal of granulosa cells in the process of oocyte retrieval leads to depletion of the cell pool needed to produce progesterone in the luteal phase. In addition, because most IVF cycles currently involve administration of GnRH modulators, either agonists or antagonists, physiologic pulsatility necessary for adequate corpus luteum function and progesterone production is disrupted leading to well-described luteal phase foreshortening and dysfunction. $^{2-5}$ Exogenous supplementation of the luteal phase of IVF cycles with either progesterone or human chorionic gonadotropin (hCG) has been shown to result in better pregnancy rates and outcomes compared with no supplementation. ${ }^{6,7}$ However, data relative to the best method of luteal phase support in IVF have been insufficient until recently. ${ }^{6}$ The best method for luteal phase support in IVF should be optimally efficacious, with minimal side effects and with ease and convenience of administration. A 2011
Issue Theme Best Practices in In Vitro Fertilization; Guest Editor, Bradley J. Van Voorhis, MD
Copyright (c) 2015 by Thieme Medical Publishers, Inc., 333 Seventh Avenue, New York, NY 10001, USA. Tel: +1(212) 584-4662.
DOI http://dx.doi.org/ 10.1055/s-0035-1545363. ISSN 1526-8004. 
Cochrane systematic review of 69 studies comparing vaginal progesterone supplementation, intramuscular progesterone supplementation, and supplementation with hCG injections reported similar IVF pregnancy rates, but the odds for OHSS complication was 20 -fold higher with hCG than with progesterone supplementation. ${ }^{7}$ They concluded that progesterone should be the preferred form of luteal phase support in IVF over hCG injections. Oral micronized progesterone preparations that would be most convenient and easy for patients to self-administer have been shown to lack adequate efficacy. ${ }^{8,9}$ Oral synthetic progesterone preparations (dydrogesterone, chlormadinone acetate) that are available in Europe and Asia, but not in the United States, have been shown in several studies conducted in Iran, India, and Japan to have equal efficacy to vaginal micronized progesterone and to intramuscular progesterone. ${ }^{10-13}$ Although oral dydrogesterone is widely available and frequently used for a variety of gynecologic indications in Europe, there have been no reports from Europe so far on its use for luteal support in IVF, or its safety in early pregnancy. The vast majority of IVF cycles are performed in Europe and the United States where parenteral progesterone preparations have been the standard of care for decades. The question of the best parenteral progesterone preparation with respect to efficacy, convenience, and tolerability has been the focus of intense investigation in the recent years.

\section{What Is Evidence and How should We Interpret It for Our Practice?}

Practice of medicine in any field has not always been and is not always based on solid scientific data. This has not been due to the lack of desire on the part of physicians to base their decisions on the best scientific methods and evidence, but rather due to the paucity of data derived from rigorously designed and properly powered studies. The strongest evidence is derived from prospective, randomized, double-blind, placebo-controlled trials involving homogeneous patient populations with adequate numbers to have the power to detect real differences in outcomes. Under ideal circumstances confirming results from more than one such study should provide the best evidence for changing or adjusting clinical practice. $^{14}$ Prospective, randomized, adequately powered studies require substantial time and resources to complete, and the nature of some investigations often preclude the double-blind design. Smaller prospective, randomized studies but with lower powers to detect the effect of interest may be combined into meta-analyses to increase the overall validity of evidence. However, even results of the best designed studies and meta-analyses must be evaluated critically with respect to potential confounders, applicability to specific populations, and publication bias when clinical practice changes are in question. ${ }^{15}$ Although retrospective studies are relatively inexpensive and easy to complete, we should exercise great caution in interpreting data derived from retrospective reviews and studies using nonconcurrent controls because bias and confounding that are inherent in such studies produce results with limited internal or external validity. ${ }^{16}$ A good example of such retrospective studies on the subject of progesterone support in IVF with either intramuscular or vaginal progesterone preparations producing diametrically opposite conclusions are those of Papaleo et al $^{17}$ and Ho et al. ${ }^{18}$

With the issues of proper methodology in mind, we will examine the current evidence on the parenteral progesterone support in vitro fertilization-embryo transfer (IVF-ET) cycles with the main focus on prospective, randomized studies and meta-analyses.

\section{Evolution and Accumulation of Evidence on Progesterone Support in IVF}

The most common form of progesterone that has been used for luteal phase support in IVF has been progesterone in oil administered as intramuscular injections (IMP) at doses of 50 to $100 \mathrm{mg}$ daily. Though reasonably effective, intramuscular injections are painful for the patients, inconvenient to administer, usually requiring another person to help with administration, and associated with severe side effects, such as welts, infections, abscesses, allergic reactions, and even pulmonary complications requiring hospital admissions. ${ }^{19-21}$ Not surprisingly, clinicians were interested in identifying other forms of progesterone support in IVF that will be at least equally efficacious and better tolerated by patients. Belgian researchers led the way in investigations of luteal phase physiology, pathology, and corrective supplementation options related to optimal endometrial maturation and improved implantation in IVF cycles in the early 1990 s. $^{22,23}$ Using histopathologic evaluations, they demonstrated that similar and appropriate luteal endometrial maturation can be achieved with either intramuscular progesterone 100-mg daily injections, or with vaginal micronized progesterone administration at doses between 300 and $600 \mathrm{mg}$ daily. Oral micronized progesterone was ineffective in inducing secretory endometrial changes. ${ }^{24}$ With this information on hand, Smitz et $\mathrm{al}^{25}$ set out a first prospective randomized study comparing pregnancy rates in IVF cycles supplemented with either intramuscular progesterone in oil or with micronized progesterone in vaginal capsules. They randomized 262 IVF, gamete intrafallopian transfer (GIFT), and zygote intrafallopian transfer (ZIFT) patients into two groups to receive either intramuscular progesterone injections $50 \mathrm{mg}$ daily, or micronized progesterone in capsules vaginally $600 \mathrm{mg}$ in three divided doses starting on the day before oocyte retrieval. They also measured luteal phase and early pregnancy serum progesterone levels in pregnant and nonpregnant patients, and found that despite significantly higher serum progesterone levels observed in patients receiving intramuscular progesterone, those receiving vaginal progesterone had a higher overall pregnancy rate as well as implantation rate.

Although original histopathologic studies ${ }^{24,26}$ demonstrating adequate secretory endometrial maturation used micronized progesterone capsules at doses between 300 and $600 \mathrm{mg}$ daily, several randomized studies comparing vaginal and intramuscular progesterone supplementation in IVF in the mid-to-late 1990s chose to use lower vaginal 
doses (100-200 mg daily) and in different and nonstandardized compounded preparations such as creams and suppositories. $^{27-29}$ Such doses and preparations are currently recognized as insufficient and inappropriate for luteal phase support in IVF.

The first meta-analysis of luteal phase support in infertility treatment that addressed comparison of vaginal versus intramuscular progesterone ${ }^{30}$ preparations included five prospective studies two of which ${ }^{27,28}$ contained the majority of patients included in the meta-analysis. Those two studies used vaginal progesterone preparations of 100 to $200 \mathrm{mg}$ daily. The authors concluded that intramuscular progesterone conferred the most benefit compared with vaginal route. It is implicitly understood that the value of conclusions that can be drawn from any meta-analysis is dependent on the size and study design quality of its individual components. ${ }^{14}$ It is not surprising therefore that a meta-analysis that included the majority of patients receiving insufficient amounts of vaginal progesterone for luteal support would come to an unfavorable conclusion.

By the time Daya and Gunby ${ }^{31}$ published their metaanalysis in 2004, five additional prospective studies comparing vaginal and intramuscular progesterone preparations had been eligible for inclusion. Only 2 out of 10 studies reported live births as an outcome, and statistical significance was reached in favor of intramuscular progesterone administration for the live birth parameter. However, authors of one of those two studies admitted to methodological flaws related to lack of stratification by age leading to a greater number of patients older than 40 years in the vaginal progesterone group, thus biasing results by the most important confounders of IVF outcome-age and ovarian reserve. ${ }^{32}$

Given continued uncertainly of the comparative efficacy of intramuscular versus vaginal progesterone supplementation in IVF, there was a great need for rigorously designed and properly powered prospective randomized studies using proper therapeutic progesterone doses to resolve the issue. Two studies that fulfilled these requirements were published in $2008 .^{33,34}$ Both studies aimed at elimination of important confounders such as age and ovarian reserve by excluding patients older than 40 years, and those with day 3 folliclestimulating hormone (FSH) levels greater than $15 \mathrm{mIU} / \mathrm{mL}$. Dal Prato et $\mathrm{al}^{33}$ restricted enrollment to women younger than 37 years, and Yanushpolsky et $\mathrm{al}^{34}$ stratified women by age younger than 35 years and 35 to 39 years old prior to randomization to ensure equal age distribution between treatment groups. Micronized vaginal progesterone bioadhesive gel (Crinone $8 \%$ [90 mg] vaginal gel) was the first commercially available, FDA-approved preparation for progesterone supplementation at once daily dose, and for replacement at twice per day dosing in IVF treatments. Excellent transvaginal absorption and superior progesterone tissue levels achieved with Crinone $8 \%$ as compared with intramuscular progesterone were well demonstrated in several prior studies. ${ }^{35-37}$ Dal Prato et $\mathrm{al}^{33}$ presented a noninferiority study design for three luteal phase treatment arms in IVF patients: intramuscular progesterone $50 \mathrm{mg} /$ day, Crinone $8 \%$ (90 mg) vaginal gel once a day, and Crinone $8 \%$ vaginal gel twice a day starting the day after oocyte retrieval. They achieved their precalculated necessary sample size with 138 patients in the intramuscular progesterone group, 137 patients in Crinone $8 \%$ once daily group, and 137 patients in the Crinone $8 \%$ twice daily administration group. No statistical differences were found between the three groups with respect to pregnancy rates (hCG $>5 \mathrm{mIU} / \mathrm{mL}$ ), implantation rates, clinical pregnancy rates, miscarriages and delivery rates. The authors concluded that vaginal gel can be successfully used as an alternative to intramuscular progesterone in luteal support in IVF, and that once-daily dose was sufficient for optimal results.

A two-arm comparison study by Yanushpolsky et $\mathrm{al}^{38}$ randomized 468 patients to receive either intramuscular progesterone supplementation starting the day after oocyte retrieval or Crinone $8 \%$ vaginal gel starting 2 days after oocyte retrieval. The rationale for a later start of Crinone was based on the known superior absorption data, ${ }^{35-37}$ as well as favorable results reported in preliminary studies by Schoolcraft et al. ${ }^{39}$ Similar results were observed between intramuscular progesterone and vaginal progesterone arms with respect to positive $\beta$-hCG rates, implantation rates, and clinical and ongoing/delivered pregnancy rates, as well as failed pregnancies. In follow-up questionnaires patients expressed significantly greater satisfaction with vaginal route of progesterone administration. ${ }^{38}$

The most recent and rigorous meta-analysis on the route of luteal progesterone administration in $\mathrm{IVF}^{40}$ included both Dal Prato et al's ${ }^{33}$ and Yanushpolsky et al's ${ }^{34}$ studies with proper methodology and dosing, and excluded three studies with subtherapeutic progesterone doses. ${ }^{27-29}$ A total of nine studies met inclusion criteria with seven of nine using Crinone $8 \%$ vaginal gel and two using micronized progesterone capsules $600 \mathrm{mg}$ (200 mg thrice a day). All studies used intramuscular progesterone in oil $50 \mathrm{mg}$ daily. The endpoints of analysis were clinical pregnancy and ongoing pregnancy rates. The odds ratio for these endpoints were 0.91 (95\% confidence interval [CI]: $0.74-1.13$ ), and 0.94 (95\% CI: 0.71-1.26). The authors concluded that daily administration of vaginal progesterone (90 mg bioadhesive gel or $600 \mathrm{mg}$ [200 mg thrice a day] progesterone capsules) is comparable to daily administration of $50 \mathrm{mg}$ intramuscular progesterone in oil for luteal phase support in IVF. It should be noted that all publications included in the meta-analysis reported use of "long" GnRH downregulation regimens for patients enrolled in the studies.

Kahraman and colleagues ${ }^{41}$ conducted the first prospective, randomized study of progesterone formulations for luteal support in IVF patients on GnRH-antagonist protocols. This study was adequately powered at 209 and 217 patients in each of the treatment arms and important confounders were properly excluded. Older patients ( $>37$ years old), those with diminished ovarian reserve according to their basal antral follicle counts and FSH levels, as well as patients who failed in at least three prior IVF cycle, were excluded from enrollment. Eligible patients were randomized into two groups to receive either intramuscular progesterone $100 \mathrm{mg}$ daily, or Crinone $8 \%$ (90 mg) gel twice daily on the day after oocyte retrieval. Progesterone supplementation was extended up to the evidence of fetal heart activity on ultrasound examination. 
Investigators found similar implantation, pregnancy, and clinical and ongoing pregnancy rates, as well as biochemical and miscarriage rates in both arms. The authors admitted that the rationale for using vaginal gel twice daily was not based on solid data, as they were aware of the data demonstrating equal efficacy of once- versus twice-daily regimens, ${ }^{33}$ but more out of desire to reduce possible patient anxiety.

Two other parenteral progesterone preparations for luteal support in IVF have been recently investigated and compared with established vaginal preparations. Progesterone vaginal ring (Milprosa) was compared with $8 \%$ Crinone vaginal gel in a randomized multicenter noninferiority trial led by Stadtmauer. $^{42}$ The study was conducted in 22 clinical sites in the United States in which 646 patients were randomized to once a week insertion of Progesterone vaginal ring and 651 patients received daily inserts of $8 \%$ Crinone vaginal gel starting on the day after oocyte retrieval. There were no statistical differences in pregnancy rates, live-birth rates, or major complications between treatment groups. Patient satisfaction rates and preferences were not reported in the publication, but a threefold higher rate of vaginal discharge with progesterone vaginal ring compared with vaginal gel was recorded in the Adverse Effect section. Progesterone vaginal ring for luteal phase support in IVF is not yet available on the market.

A novel aqueous subcutaneous lyophilized progesterone preparation (Prolutex) was recently compared with vaginal progesterone inserts (Endometrin) in a randomized multicenter, noninferiority study. ${ }^{43}$ A total of 800 women between ages 18 and 42 from eight U.S. centers were randomized to receive either subcutaneous aqueous progesterone injection (25 mg daily), or vaginal progesterone inserts (100 mg twice daily) starting on the day of oocyte retrieval. The results of the trial were consistent with a noninferiority of subcutaneous progesterone for luteal phase support compared with vaginal inserts and both preparations were well tolerated by the patients. The authors suggested that some patients may prefer subcutaneous route of progesterone administration over vaginal route, but actual patients' preferences had not been recorded or reported in the published manuscript. Aqueous subcutaneous progesterone solution is currently available as a ready-to-use solution in some European countries.

In summary, current body of scientific evidence confirms equal efficacy of intramuscular and vaginal progesterone preparations in therapeutic doses for luteal phase support. Vaginal progesterone preparations are more convenient and better tolerated by patients than intramuscular preparations and therefore should be the first line of progesterone supplementation in IVF. The roles of novel parenteral progesterone preparations, which are not yet widely commercially available, as well as synthetic oral progestins for luteal phase support in IVF, await confirmation in additional randomized trials that also record patient preferences.

Several other aspects of luteal phase support deserve discussion:

1. What is the optimal dose of progesterone?

2. How do various vaginal progesterone preparations compare with respect to efficacy and acceptance by patients?
3. What is the optimal timing of progesterone initiation within the IVF cycle?

4. How long should progesterone be continued in early pregnancy, and should we monitor serum progesterone levels?

5. What is the optimal luteal phase support in GnRH-agonist-triggered cycles?

6. Which progesterone preparation should be used for replacement in frozen embryo transfer cycles and donor oocyte recipient cycles?

We address each of these questions separately with respect to the evidence available at this time.

\section{What Is the Optimal Dose of Vaginal Progesterone Supplementation?}

An appropriate dose of any medication for any indication should be the dose that will be optimally effective, most convenient to administer, as well as cost-effective. The body of literature that we have reviewed so far focused on comparison of intramuscular progesterone preparations from 50 to $100 \mathrm{mg}$ daily with intravaginal preparations of micronized progesterone in oil capsules ranging from 100 to $600 \mathrm{mg}$ daily in three divided doses, or micronized progesterone bioadhesive gel (Crinone 8\% [90 $\mathrm{mg}]$ ) once or twice daily. It has been well demonstrated in histopathologic studies, ${ }^{26}$ as well as clinical studies, ${ }^{25}$ that progesterone capsules in doses less than $300 \mathrm{mg} /$ day are inferior in efficacy to intramuscular progesterone. Most studies demonstrating equal efficacy involved micronized progesterone capsules $600 \mathrm{mg}$ (200 mg thrice a day).

Crinone vaginal gel has been shown to have an excellent coefficient of absorption ${ }^{35-37}$ into endometrial tissues with ability to induce appropriate secretory endometrial changes necessary for luteal phase support. ${ }^{39}$ A single daily dose $(90 \mathrm{mg}$ ) was approved by FDA for progesterone supplementation in assisted reproductive technology (ART), whereas twice-daily dose was recommended for replacement in postmenopausal or agonadal women. ${ }^{44,45}$ Among prospective randomized studies comparing intramuscular progesterone to Crinone gel that demonstrated similar results, some involved once-daily gel administration, whereas others involved twice-daily doses. ${ }^{38,41} \mathrm{~A}$ study by Dal Prato el al contained three arms comparing intramuscular progesterone $50 \mathrm{mg}$ daily, with Crinone gel once a day and Crinone gel twice a day. All three arms had similar results on IVF outcomes. ${ }^{33}$

We conclude that a safely effective dose of micronized progesterone in oil capsules is $600 \mathrm{mg}$ daily (200 $\mathrm{mg}$ thrice a day), whereas both single-dose and as well as twice-daily dose of Crinone $8 \%$ gel are efficacious for luteal phase support.

For convenience, single-dose medications are preferred by patients. Total costs have to be compared between preparations and weighed against convenience, which is a choice that each patient will make for herself. 


\section{How Do Various Vaginal Progesterone Preparations Compare with Respect to Efficacy and Acceptance by Patients?}

There are currently three standardized vaginal progesterone formulations commercially available for use in IVF. Micronized progesterone capsules (Utrogestan/Prometrium) are available and widely used in Europe and South America, but not FDA approved for use in IVF/ART in the United States. The two progesterone formulations approved by FDA for use in IVF are Crinone 8\% vaginal gel and Endometrin $100 \mathrm{mg}$ (natural progesterone in capsules). The unique feature of Crinone bioadhesive gel is its property of sustained-release delivery over time allowing for once-daily administration. Other formulations require multiple daily doses for optimal efficacy.

Though both Utrogestan and Crinone have been compared in prospective, randomized trials to intramuscular progesterone and have been shown to be of equal efficacy in appropriate doses (as discussed previously), no such comparison exists for Endometrin. Instead, Endometrin at twice- and thrice-daily doses has been compared with Crinone oncedaily dose for luteal support in IVF in a prospective, randomized multicenter trial with non-inferiority design. ${ }^{46}$ The study had enough power to demonstrate noninferiority of Endometrin at twice a day and thrice a day doses in women younger than 35 years with FSH less than $10 \mathrm{mIU} / \mathrm{mL}$ compared with Crinone $8 \%$ gel once daily. The authors stated that the study did not reach enough power for meaningful conclusions for women older than 35 .

Three other prospective studies addressed comparison of micronized progesterone capsules (Utrogestan $600 \mathrm{mg}$ ) in two or three doses a day with Crinone gel once daily, ${ }^{47-49}$ and Simunic et $\mathrm{al}^{47}$ also assessed patient preference for either of the preparations. All three studies concluded that pregnancy rates were similar between Utrogestan and Crinone groups, but patients reported superior tolerability and acceptability of Crinone in the study of Simunic et al.

A recent meta-analysis ${ }^{50}$ pooled together results of seven randomized trials comparing once- or twice-daily Crinone vaginal gel with either twice- or thrice-daily Endometrin or thrice-daily Utrogestan. The authors concluded that all vaginal progesterone preparations in adequate doses were equally efficacious for luteal support in IVF cycles with respect to clinical pregnancy rates.

We conclude that all three available vaginal progesterone preparations-Crinone gel once a day, Utrogestan $200 \mathrm{mg}$ thrice a day, and Endometrin $100 \mathrm{mg}$ twice or thrice a dayare equally effective for luteal phase support in IVF cycles. However, data are insufficient for definite conclusion on the efficacy of Endometrin in women older than 35.

\section{What Is the Optimal Timing of Progesterone Initiation within the IVF Cycle?}

In stimulated IVF cycles there is substantial endogenous progesterone production starting immediately after, and possibly even slightly before, the hCG trigger. Though proges- terone supplementation in the luteal phase is important, it is similarly important not to advance endometrial maturation out of synch with embryo development. It has been demonstrated that administration of intramuscular progesterone early in the cycle, that is, prior to oocyte retrieval compared with within 24 hours after oocyte retrieval, will result in lower pregnancy rates. ${ }^{51}$ Mochtar et $\mathrm{al}^{52}$ examined effects of timing of vaginal progesterone initiation (micronized progesterone $400 \mathrm{mg}$ twice daily) in a prospective randomized design $(n=385)$. Progesterone was initiated either on the day of hCG trigger, on the day of oocyte retrieval, or on the day of embryos transfer (day 3). Though ongoing pregnancy rate was lower in the hCG group compared with two others, the difference was not statistically significant. If starting progesterone supplementation too early in the cycle may have a negative effect on the outcome, starting progesterone too late could be equally detrimental. A prospective, randomized study by Williams et $\mathrm{al}^{53}$ comparing progesterone start 3 versus 6 days after retrieval found higher pregnancy rates with earlier start. There was heterogeneity with the exact timing of progesterone start in prospective studies that we examined earlier in this article. Dal Prato et al, ${ }^{33}$ Kahraman et $\mathrm{al}^{41}$ and Doody et $\mathrm{al}^{46}$ started supplementation on the day after retrieval, whereas Yanushpolsky et $\mathrm{al}^{38}$ and a smaller trial by Schoolcraft et $\mathrm{al}^{39}$ started supplementation with vaginal progesterone 2 days after retrieval. All studies had similar results.

We conclude that there is an acceptable window of time24 to 48 hours after oocyte retrieval for initiation of progesterone supplementation with optimal cycle results.

\section{How Long Should Progesterone Be Continued in Early Pregnancy and Do We Need to Monitor Serum Progesterone Levels?}

Though it has long been established that luteal phase support with progesterone in IVF cycles leads to improved outcomes, there are still limited data and little consensus on the necessary duration for progesterone supplementation in early pregnancy, that is, beyond first positive pregnancy test. Nyboe Andersen et $\mathrm{al}^{54}$ conducted a prospective study in which patients enrolled in stimulated IVF cycles and receiving vaginal micronized progesterone $600 \mathrm{mg}$ in the luteal phase were randomized to stopping progesterone support after first diagnosis of pregnancy versus continuing for 3 more weeks $(n=303)$. They found no differences in miscarriage rates and argued that progesterone supplementation can be safely withdrawn after the first positive $\beta$-hCG result.

Aboulghar and colleagues ${ }^{55}$ conducted a survey of 21 IVF programs on progesterone support in early pregnancy after IVF. They found no uniform pattern or consensus, and then set out a prospective study in which they randomized patients to either continuation or discontinuation of progesterone support on the day of first ultrasound demonstrating positive fetal heart activity $(n=257)$. They found no significant differences in miscarriage rates, or bleeding in early pregnancy between the groups. They concluded that there was no 
advantage to continuing progesterone support beyond the time of first ultrasound viability study.

Three other smaller randomized studies comparing early versus late cessation of progesterone supplementation in early pregnancy ${ }^{56-58}$ were combined with the studies by Nyboe Andersen $^{54}$ and Aboulghar ${ }^{55}$ in a meta-analysis evaluating the optimal duration of progesterone supplementation in pregnant women after IVF/ICSI. ${ }^{59}$ The authors concluded that currently available evidence suggests that progesterone supplementation beyond the first positive hCG test after IVF/ICSI might generally be unnecessary. They called for large, prospective, randomized trials to settle the issue of duration of progesterone support in early IVF pregnancies. Because the first group of studies by Smitz and Devroy $^{25}$ showing lower serum progesterone levels but adequate endometrial maturation, and similar pregnancy rates with vaginal progesterone preparations compared with intramuscular preparations, there has been little reason to be concerned with serum progesterone level monitoring. In addition, absorption and pharmacokinetic studies by Bulletti ${ }^{35}$ and Cicinelli ${ }^{36}$ confirmed that there was no correlation between the desired endometrial maturation effect and serum progesterone levels, and that there is no reason to subject patients to unnecessary blood drawings, and potentially unsubstantiated reasons for anxiety involved with serum level monitoring. None of the major randomized studies that demonstrated equal efficacy of vaginal and intramuscular preparations reported serum progesterone levels because they were felt to be irrelevant to the outcomes. $^{33,38,41}$

There is no scientific evidence for monitoring serum progesterone levels during supplemented luteal phase of stimulated IVF cycles.

\section{What Is the Optimal Luteal Phase Support in GnRH-Agonist-Triggered Cycles?}

All previously reviewed data on luteal phase support were in IVF cycles in which final oocyte maturation was triggered with either hCG in doses between 5,000 and 10,000 IU or with recombinant-hCG (rhCG) in doses between 250 and $500 \mu \mathrm{g}$. Half-life of hCG has been estimated at 2.3 days and serum hCG levels are known to be detectable by immunoenzymetric up to 14 days after injection. ${ }^{60}$ This means that in addition to effecting the final oocyte maturation, hCG also provides luteal phase support that contributes to the increased risk of developing OHSS. ${ }^{61}$ OHSS has been observed even in oocyte donors whose only source of hCG could be the trigger injection, not endogenous hCG related to pregnancy. In IVF cycles in which pituitary suppression is achieved with $\mathrm{GnRH}$ antagonists, there is an option to effect final oocyte maturation with more physiologic GnRH-agonist triggers instead of hCG. Galindo et $\mathrm{al}^{62}$ randomized 212 oocyte donors to receive either GnRH-agonist (Triptorelin $0.2 \mathrm{mg}$ ) trigger or rhCG $(250 \mu \mathrm{g})$, and demonstrated similar oocyte maturity and yield between the groups, and similar implantation rates and ongoing pregnancy rates among recipients. None of the oocyte donors triggered with GnRH agonist developed OHSS, whereas 10 of 106 donors triggered with rhCG developed OHSS symptoms. However, significantly lower ongoing pregnancy rates were observed when GnRH agonist was used to trigger autologous IVF/ET cycles in a randomized study by Kolibianakis et al. ${ }^{63}$ Humaidan and colleagues $^{64}$ hypothesized that a shorter half-life of an LH surge induced by GnRH-agonist trigger may not be providing sufficient luteotrophic effect necessary for optimal endometrial receptivity in GnRH-antagonist-controlled cycles. They conducted a randomized clinical trial in which 152 patients received GnRH-agonist trigger and also hCG $1,500 \mathrm{IU} / \mathrm{IM}$ on the day of oocyte retrieval. In the other arm 150 patients were triggered with hCG 10,000 IU/SC. Delivery rates of 24 and $31 \%$, respectively, were observed and were not statistically different. None of the patients in the GnRHagonist/hCG group developed OHSS, whereas three patients in the hCG group had OHSS symptoms. The authors suggested that modified luteal phase support protocols that include one to two small doses of hCG (1,000-1,500 IU) between the time of trigger and mid-luteal phase in addition to the standard progesterone supplementation would decrease incidence of OHSS without compromising pregnancy rates. ${ }^{65}$ Their suggestion is supported by the findings of different endometrial gene expression studies in the luteal phase of oocyte donors triggered with rhCG versus GnRH agonists and supplemented in the luteal phase with either standard vaginal progesterone alone, oral estrogen and vaginal progesterone, or with rhCG or recombinant-LH $(\mathrm{rLH})$ in addition to the oral estrogen and vaginal progesterone preparations. ${ }^{66}$ Gene expression after GnRH-agonist trigger and modified luteal support that included LH/hCG activity more closely resembled the patterns seen in the rhCG-triggered group.

Current evidence suggests that GnRH-agonist and hCGtriggered cycles result in equivalent oocyte maturity and yields, but GnRH triggers are associated with a much lower OHSS rate. Consideration should be given to triggering all cycles that do not involve fresh embryo transfers (egg donors, fertility preservation) with GnRH agonists only. GnRH-agonist-triggered cycles are characterized by a higher degree of luteal phase impairment compared with hCG-triggered cycles, but this impairment may be overcome by addition of small doses of hCG to the standard progesterone/estrogen regimen and this is not associated with significant risks of OHSS. The optimal regimen of intensive luteal phase support involving progesterone, estrogen, and hCG in GnRH-agonisttriggered cycles should be defined through a large randomized trial. ${ }^{65}$

\section{What about Progesterone Replacement in Frozen Embryo Transfer Cycles and Donor Oocyte Recipient Cycles?}

Frozen embryo transfer cycles and donor oocyte recipient cycles are different from stimulated IVF cycles in that there is no endogenous progesterone production, and therefore instead of luteal phase supplementation, there is a need for luteal phase "creation" or replacement. 
Just as with stimulated cycles intramuscular progesterone in doses of 50 to $100 \mathrm{mg}$ daily has been the most common progesterone preparation used in these replacement cycles, and just as with stimulated cycles, there have been adverse reactions, complications, and inconveniences for patients related to intramuscular progesterone administration. There is a need to find more tolerable but equally efficacious progesterone formulations for the replacement cycles. Unfortunately there is a paucity of data addressing comparison of intramuscular and vaginal progesterone preparations in frozen embryo transfer and donor egg/recipient cycles, and no definite conclusions can be drawn at this time. Crinone vaginal gel has been approved by FDA for supplementation at once-daily dose and for replacement at twice-daily dose. There are only two small prospective studies comparing intramuscular progesterone to Crinone vaginal gel twice a day and once a day in donor egg/recipient cycles with similar results. ${ }^{45,67}$ Three reports by Berger et al and Williams et $\mathrm{al}^{68-70}$ are retrospective studies also showing equivalent pregnancy outcomes for frozen and donor egg/recipient cycles in intramuscular progesterone and Crinone vaginal gel groups at twice-daily doses as well as once-daily doses. A large retrospective cohort study comparing intramuscular progesterone with at a 50 - to $100-\mathrm{mg}$ daily dose $(n=440)$ and Crinone vaginal gel $90 \mathrm{mg}$ twice a day $(n=298)$ in frozen cycles (FET) with day 3 embryos showed significantly higher clinical pregnancy and live-birth rates in cycles replaced with intramuscular progesterone. ${ }^{71}$ However, an even larger retrospective cohort study ${ }^{72}$ compared intramuscular progesterone $50 \mathrm{mg}$ daily $(n=682)$ and Crinone vaginal gel $90 \mathrm{mg}$ twice a day $(n=238)$ in FET cycles in which only vitrified blastocysts were transferred and found no differences in implantation, clinical pregnancy, or live-birth rates between the groups. As all retrospective studies, these cannot be relied on as definite evidence of efficacy or lack thereof. Adequately powered and properly designed prospective, randomized trials are much needed to establish efficacy of vaginal progesterone replacement in frozen embryo transfer cycles and donor egg/recipient cycles.

\section{Is There Benefit of Adding Estradiol to Progesterone for Support of the Luteal Phase in the IVF Cycles?}

The pattern of estrogen and progesterone decline in the luteal phase of IVF cycles has been well described in the literature and lower pregnancy rates have been observed with lower luteal phase estradiol to progesterone ratios. ${ }^{73,74}$ The question of causality versus association, that is, do the lower estradiol levels in the luteal phase cause lower pregnancy rates, or are lower estradiol levels the result of lack of implantation, could not be resolved without properly designed studies. Several small prospective randomized studies addressing addition of estradiol supplementation in doses between 2 and $6 \mathrm{mg} /$ day suggested a benefit, whereas almost a similar number of small prospective randomized studies demonstrated no improvement in pregnancy rates with estradiol supplementation. ${ }^{6}$ In 2008 , Kolibianakis et $\mathrm{al}^{75}$ published a systematic review and meta-analysis of four prospective randomized studies ( $n=587$ patients) addressing IVF cycle outcomes of patients who received estradiol supplementation in the luteal phase and those who did not. They concluded that the evidence available at the time suggested that the addition of estrogen to progesterone for luteal phase support does not increase the probability of pregnancy in IVF. A more recent meta-analysis of nine randomized studies presented by Jee et $\mathrm{al}^{76}$ showed no statistically significant differences in IVF pregnancy rates between the groups of patients who received additional estradiol in the luteal phase and those who received progesterone supplementation alone. Authors of both meta-analyses called for large, prospective randomized studies to confirm their findings.

A prospective randomized study was subsequently published by Elgindy et al. ${ }^{77}$ It included 270 patients undergoing ICSI cycles in long agonist protocols in three arms. All patients received intramuscular progesterone (100 mg daily). Patients in group $A$ received no additional estrogen, whereas patients in group B received additional oral estradiol valerate $6 \mathrm{mg}$ daily, and patients in group $C$ received daily estradiol valerate $6 \mathrm{mg}$ per vagina. There were no differences in progesterone levels in the luteal phase among all three groups, and luteal estradiol levels were similar between groups B and C. Likewise, there were no differences in pregnancy rates between progesterone only group (group A), and oral estradiol supplemented group (group B), but higher pregnancy rates were observed in patients supplemented with vaginal estradiol valerate $6 \mathrm{mg}$ daily. The authors concluded that pregnancy rates in patients undergoing ICSI in long agonist protocols may be improved by addition of vaginal estradiol in the luteal phase.

In 2013, Lin et al ${ }^{78}$ published the largest prospective study so far that included 402 patients undergoing IVF or ICSI cycles with GnRH-agonist suppression. Patients were randomized into two groups. The study group $(n=202)$ received intramuscular progesterone $(60 \mathrm{mg} /$ day) plus oral estradiol valerate $(3 \mathrm{mg}$ bid) whereas control group $(n=200)$ received intramuscular progesterone alone $(60 \mathrm{mg} / \mathrm{day})$ for luteal support. There were no differences between the groups with respect to pregnancy rate, live-birth rate, or miscarriage rate.

The preponderance of data demonstrates that oral estrogen supplementation in the luteal phase does not improve outcomes of IVF/ICSI cycles. The answer to the question of whether vaginal estrogen supplementation perhaps in combination with oral or subcutaneous progesterone preparations may improve IVF outcomes will have to await completion of adequately powered prospective randomized studies.

\section{Conclusion}

1. Progesterone supplementation of luteal phase of stimulated IVF cycles leads to higher pregnancy rates and is necessary for optimal results. Vaginal progesterone 
supplementation is equally effective and better tolerated by patients than intramuscular preparations. Sufficient evidence of efficacy and tolerability to make vaginal supplementation the main route of progesterone support in stimulated IVF cycles exists.

2. Once-daily Crinone gel, or twice- or thrice-daily Endometrin, or micronized progesterone $200 \mathrm{mg}$ thrice a day are optimal doses of vaginal progesterone for luteal phase support in IVF. Patients should have a choice about which preparation to use based on convenience and cost considerations.

3. A window of time exists when vaginal progesterone should be initiated for optimal pregnancy results. Vaginal supplementation is best started within 24 to 48 hours after oocyte retrieval.

4. There is no need for serum progesterone level monitoring with vaginal supplementation as little correlation exists between serum levels and local endometrial effects or pregnancy outcomes.

5. It is likely that continued progesterone support in early pregnancy beyond the first positive pregnancy test is of little value for successful outcome; however, there are insufficient data to date to establish the right time for discontinuing support. Large randomized, prospective studies are needed to establish parameters for discontinuation, but they may be complicated by difficulty in subject recruitment secondary to patients' anxiety.

6. IVF cycles that do not involve fresh embryo transfers (oocyte donor cycles, fertility preservation cycles) may be best conducted with GnRH-antagonist pituitary suppression and $\mathrm{GnRH}$-agonist trigger to minimize the risk of OHSS without compromising oocyte yield and quality.

7. GnRH-antagonist cycles that are triggered with $\mathrm{GnRH}$ agonists and that involve fresh embryo transfers should have a modified luteal phase support regimen that includes small doses of hCG or LH preparations in addition to standard progesterone support for optimal outcomes.

8. Current evidence comparing progesterone replacement in frozen embryo transfer cycles and donor egg/recipient cycles with either traditional intramuscular progesterone or vaginal progesterone is based on heterogeneous retrospective studies only and is insufficient to determine the optimal protocol with respect to efficacy and patient preference. Large, prospective, randomized studies are needed to resolve this issue.

9. Addition of oral estradiol to progesterone supplementation in the luteal phase of IVF cycles does not improve outcomes. More studies are needed to evaluate potential benefits of vaginal estradiol supplementation.

10. New developments related to subcutaneous and oral synthetic progesterone preparations deserve careful attention.

\section{References}

1 Filicori M, Butler JP, Crowley WF Jr. Neuroendocrine regulation of the corpus luteum in the human. Evidence for pulsatile progesterone secretion. J Clin Invest 1984;73(6):1638-1647
2 Yanushpolsky E. Evidence-based use of progesterone in IVF. In: Racowsky C, Carrell DT, Schlegel PN, Fauser BC, eds. Biennial Review of Infertility (Volume 2). New York, NY: Springer; 2011: 79-90

3 Jones GS, Garcia J, Acosta A. Luteal phase evaluation in in-vitro fertilization. In: Edwards RG, Purdy JM, eds. Human Conception In Vitro. London, UK: Academic Press; 1982:297-310

4 Tavaniotou A, Devroey P. Luteal hormonal profile of oocyte donors stimulated with a GnRH antagonist compared with natural cycles. Reprod Biomed Online 2006;13(3):326-330

5 Beckers NG, Macklon NS, Eijkemans MJ, et al. Nonsupplemented luteal phase characteristics after the administration of recombinant human chorionic gonadotropin, recombinant luteinizing hormone, or gonadotropin-releasing hormone $(\mathrm{GnRH})$ agonist to induce final oocyte maturation in in vitro fertilization patients after ovarian stimulation with recombinant follicle-stimulating hormone and GnRH antagonist cotreatment. J Clin Endocrinol Metab 2003;88(9):4186-4192

6 Hubayter ZR, Muasher SJ. Luteal supplementation in in vitro fertilization: more questions than answers. Fertil Steril 2008; 89(4):749-758

7 van der Linden M, Buckingham K, Farquhar C, Kremer JA, Metwally M. Luteal phase support for assisted reproduction cycles. Cochrane Database Syst Rev 2011;(10):CD009154

8 Licciardi FL, Kwiatkowski A, Noyes NL, Berkeley AS, Krey LL, Grifo JA. Oral versus intramuscular progesterone for in vitro fertilization: a prospective randomized study. Fertil Steril 1999;71(4): 614-618

9 Friedler S, Raziel A, Schachter M, Strassburger D, Bukovsky I, RonEl R. Luteal support with micronized progesterone following invitro fertilization using a down-regulation protocol with gonadotrophin-releasing hormone agonist: a comparative study between vaginal and oral administration. Hum Reprod 1999;14(8): 1944-1948

10 Salehpour S, Tamimi M, Saharkhiz N. Comparison of oral dydrogesterone with suppository vaginal progesterone for luteal-phase support in in vitro fertilization (IVF): a randomized clinical trial. Iran J Reprod Med 2013;11(11):913-918

11 Ganesh A, Chakravorty N, Mukherjee R, Goswami S, Chaudhury K, Chakravarty B. Comparison of oral dydrogestrone with progesterone gel and micronized progesterone for luteal support in 1,373 women undergoing in vitro fertilization: a randomized clinical study. Fertil Steril 2011;95(6):1961-1965

12 Iwase A, Ando H, Toda S, et al. Oral progestogen versus intramuscular progesterone for luteal support after assisted reproductive technology treatment: a prospective randomized study. Arch Gynecol Obstet 2008;277(4):319-324

13 Patki A, Pawar VC. Modulating fertility outcome in assisted reproductive technologies by the use of dydrogesterone. Gynecol Endocrinol 2007;23(Suppl 1):68-72

14 Scifres CM, Iams JD, Klebanoff M, Macones GA. Meta-analysis vs. large clinical trials: which should guide our management? Am J Obstet Gynecol 2009;200:484.e1-484.e5

15 Timmermans S, Mauck A. The promises and pitfalls of evidencebased medicine. Health Aff (Millwood) 2005;24(1):18-28

16 Atkins D, Best D, Briss PA, et al; GRADE Working Group. Grading quality of evidence and strength of recommendations. BMJ 2004; 328(7454): 1490

17 Papaleo E, Quaranta L, Molgora M. Intramuscular vs intravaginal natural progesterone in patients undergoing in vitro fertilization-embryo transfer cycles. A retrospective observational, case-control study. Eur Rev Med Pharmacol Sci 2010;14(2): 103-106

18 Ho CH, Chen SU, Peng FS, Chang CY, Yang YS. Luteal support for IVF/ ICSI cycles with Crinone $8 \%(90 \mathrm{mg}$ ) twice daily results in higher pregnancy rates than with intramuscular progesterone. J Chin Med Assoc 2008;71(8):386-391 
19 Bouckaert Y, Robert F, Englert Y, De Backer D, De Vuyst P, Delbaere A. Acute eosinophilic pneumonia associated with intramuscular administration of progesterone as luteal phase support after IVF: case report. Hum Reprod 2004;19(8):1806-1810

20 Veysman B, Vlahos I, Oshva L. Pneumonitis and eosinophilia after in vitro fertilization treatment. Ann Emerg Med 2006;47(5):472-475

21 Phy JL, Weiss WT, Weiler CR, Damario MA. Hypersensitivity to progesterone-in-oil after in vitro fertilization and embryo transfer. Fertil Steril 2003;80(5):1272-1275

22 Van Steirteghem AC, Smitz J, Camus M, et al. The luteal phase after in-vitro fertilization and related procedures. Hum Reprod 1988; $3(2): 161-164$

23 Smitz J, Devroey P, Camus M, et al. The luteal phase and early pregnancy after combined GnRH-agonist/HMG treatment for superovulation in IVF or GIFT. Hum Reprod 1988;3(5):585-590

24 Devroey P, Palermo G, Bourgain C, Van Waesberghe L, Smitz J, Van Steirteghem AC. Progesterone administration in patients with absent ovaries. Int J Fertil 1989;34(3):188-193

25 Smitz J, Devroey P, Faguer B, Bourgain C, Camus M, Van Steirteghem AC. A prospective randomized comparison of intramuscular or intravaginal natural progesterone as a luteal phase and early pregnancy supplement. Hum Reprod 1992;7(2):168-175

26 Bourgain C, Devroey P, Van Waesberghe L, Smitz J, Van Steirteghem AC. Effects of natural progesterone on the morphology of the endometrium in patients with primary ovarian failure. Hum Reprod 1990;5(5):537-543

27 Perino M, Brigandì FG, Abate FG, Costabile L, Balzano E, Abate A. Intramuscular versus vaginal progesterone in assisted reproduction: a comparative study. Clin Exp Obstet Gynecol 1997;24(4):228-231

28 Artini PG, Volpe A, Angioni S, Galassi MC, Battaglia C, Genazzani AR. A comparative, randomized study of three different progesterone support of the luteal phase following IVF/ET program. J Endocrinol Invest 1995;18(1):51-56

29 Porcu E. Intramuscular versus vaginal progesterone in assisted reproduction. Fertil Steril 2003;80(Suppl 3):S131

30 Pritts EA, Atwood AK. Luteal phase support in infertility treatment: a meta-analysis of the randomized trials. Hum Reprod 2002;17(9):2287-2299

31 Daya S, Gunby J. Luteal phase support in assisted reproduction cycles. Cochrane Database Syst Rev 2004;(3):CD004830

32 Propst AM, Hill JA, Ginsburg ES, Hurwitz S, Politch J, Yanushpolsky EH. A randomized study comparing Crinone $8 \%$ and intramuscular progesterone supplementation in in vitro fertilization-embryo transfer cycles. Fertil Steril 2001;76(6):1144-1149

33 Dal Prato L, Bianchi L, Cattoli M, Tarozzi N, Flamigni C, Borini A. Vaginal gel versus intramuscular progesterone for luteal phase supplementation: a prospective randomized trial. Reprod Biomed Online 2008;16(3):361-367

34 Yanushpolsky E, Hurwitz S, Greenberg L, Racowsky C, Hornstein MD. Comparison of Crinone $8 \%$ intravaginal gel and intramuscular progesterone supplementation for in vitro fertilization/embryo transfer in women under age 40: interim analysis of a prospective randomized trial. Fertil Steril 2008;89(2):485-487

35 Bulletti C, de Ziegler D, Flamigni C, et al. Targeted drug delivery in gynaecology: the first uterine pass effect. Hum Reprod 1997; 12(5):1073-1079

36 Cicinelli E, de Ziegler D, Bulletti C, Matteo MG, Schonauer LM, Galantino P. Direct transport of progesterone from vagina to uterus. Obstet Gynecol 2000;95(3):403-406

37 Miles RA, Paulson RJ, Lobo RA, Press MF, Dahmoush L, Sauer MV. Pharmacokinetics and endometrial tissue levels of progesterone after administration by intramuscular and vaginal routes: a comparative study. Fertil Steril 1994;62(3):485-490

38 Yanushpolsky E, Hurwitz S, Greenberg L, Racowsky C, Hornstein M. Crinone vaginal gel is equally effective and better tolerated than intramuscular progesterone for luteal phase support in in vitro fertilization-embryo transfer cycles: a prospective randomized study. Fertil Steril 2010;94(7):2596-2599
39 Schoolcraft WB, Hesla JS, Gee MJ. Experience with progesterone gel for luteal support in a highly successful IVF programme. Hum Reprod 2000;15(6):1284-1288

40 Zarutskie PW, Phillips JA. A meta-analysis of the route of administration of luteal phase support in assisted reproductive technology: vaginal versus intramuscular progesterone. Fertil Steril 2009; 92(1):163-169

41 Kahraman S, Karagozoglu SH, Karlikaya G. The efficiency of progesterone vaginal gel versus intramuscular progesterone for luteal phase supplementation in gonadotropin-releasing hormone antagonist cycles: a prospective clinical trial. Fertil Steril 2010; 94(2):761-763

42 Stadtmauer L, Silverberg KM, Ginsburg ES, Weiss H, Howard B. Progesterone vaginal ring versus vaginal gel for luteal support with in vitro fertilization: a randomized comparative study. Fertil Steril 2013;99(6):1543-1549

43 Baker VL, Jones CA, Doody K, et al. A randomized, controlled trial comparing the efficacy and safety of aqueous subcutaneous progesterone with vaginal progesterone for luteal phase support of in vitro fertilization. Hum Reprod 2014;29(10):2212-2220

44 Gibbons WE, Toner JP, Hamacher P, Kolm P. Experience with a novel vaginal progesterone preparation in a donor oocyte program. Fertil Steril 1998;69(1):96-101

45 Jobanputra K, Toner JP, Denoncourt R, Gibbons WE. Crinone 8\% (90 $\mathrm{mg}$ ) given once daily for progesterone replacement therapy in donor egg cycles. Fertil Steril 1999;72(6):980-984

46 Doody KJ, Schnell VL, Foulk RA, et al. Endometrin for luteal phase support in a randomized, controlled, open-label, prospective invitro fertilization trial using a combination of Menopur and Bravelle for controlled ovarian hyperstimulation. Fertil Steril 2009;91(4):1012-1017

47 Simunic V, Tomic V, Tomic J, Nizic D. Comparative study of the efficacy and tolerability of two vaginal progesterone formulations, Crinone $8 \%$ gel and Utrogestan capsules, used for luteal support. Fertil Steril 2007;87(1):83-87

48 Ludwig M, Schwartz P, Babahan B, et al. Luteal phase support using either Crinone $8 \%$ or Utrogest: results of a prospective, randomized study. Eur J Obstet Gynecol Reprod Biol 2002;103(1):48-52

49 Geber S, Moreira AC, de Paula SO, Sampaio M. Comparison between two forms of vaginally administered progesterone for luteal phase support in assisted reproduction cycles. Reprod Biomed Online 2007;14(2):155-158

50 Polyzos NP, Messini CI, Papanikolaou EG, et al. Vaginal progesterone gel for luteal phase support in IVF/ICSI cycles: a meta-analysis. Fertil Steril 2010;94(6):2083-2087

51 Sohn SH, Penzias AS, Emmi AM, et al. Administration of progesterone before oocyte retrieval negatively affects the implantation rate. Fertil Steril 1999;71(1):11-14

52 Mochtar MH, Van Wely M, Van der Veen F. Timing luteal phase support in GnRH agonist down-regulated IVF/embryo transfer cycles. Hum Reprod 2006;21(4):905-908

53 Williams SC, Oehninger S, Gibbons WE, Van Cleave WC, Muasher SJ. Delaying the initiation of progesterone supplementation results in decreased pregnancy rates after in vitro fertilization: a randomized, prospective study. Fertil Steril 2001;76(6):1140-1143

54 Nyboe Andersen A, Popovic-Todorovic B, Schmidt KT, et al. Progesterone supplementation during early gestations after IVF or ICSI has no effect on the delivery rates: a randomized controlled trial. Hum Reprod 2002;17(2):357-361

55 Aboulghar MA, Amin YM, Al-Inany HG, et al. Prospective randomized study comparing luteal phase support for ICSI patients up to the first ultrasound compared with an additional three weeks. Hum Reprod 2008;23(4):857-862

56 Kohls G, Ruiz F, Martínez M, et al. Early progesterone cessation after in vitro fertilization/intracytoplasmic sperm injection: a randomized, controlled trial. Fertil Steril 2012;98(4):858-862

57 Kyrou D, Fatemi HM, Zepiridis L, et al. Does cessation of progesterone supplementation during early pregnancy in patients treated 
with recFSH/GnRH antagonist affect ongoing pregnancy rates? A randomized controlled trial. Hum Reprod 2011;26(5):1020-1024

58 Goudge CS, Nagel TC, Damario MA. Duration of progesterone-in-oil support after in vitro fertilization and embryo transfer: a randomized, controlled trial. Fertil Steril 2010;94(3):946-951

59 Liu XR, Mu HQ Shi Q, Xiao XQ, Qi HB. The optimal duration of progesterone supplementation in pregnant women after IVF/ICSI: a meta-analysis. Reprod Biol Endocrinol 2012;10:107

60 Damewood MD, Shen W, Zacur HA, Schlaff WD, Rock JA, Wallach EE. Disappearance of exogenously administered human chorionic gonadotropin. Fertil Steril 1989;52(3):398-400

61 Delvigne A, Rozenberg S. Epidemiology and prevention of ovarian hyperstimulation syndrome (OHSS): a review. Hum Reprod Update 2002;8(6):559-577

62 Galindo A, Bodri D, Guillén JJ, Colodrón M, Vernaeve V, Coll O. Triggering with HCG or GnRH agonist in GnRH antagonist treated oocyte donation cycles: a randomised clinical trial. Gynecol Endocrinol 2009;25(1):60-66

63 Kolibianakis EM, Schultze-Mosgau A, Schroer A, et al. A lower ongoing pregnancy rate can be expected when GnRH agonist is used for triggering final oocyte maturation instead of HCG in patients undergoing IVF with GnRH antagonists. Hum Reprod 2005;20(10):2887-2892

64 Humaidan P, Ejdrup Bredkjaer H, Westergaard LG, Yding Andersen C. 1,500 IU human chorionic gonadotropin administered at oocyte retrieval rescues the luteal phase when gonadotropin-releasing hormone agonist is used for ovulation induction: a prospective, randomized, controlled study. Fertil Steril 2010;93(3):847-854

65 Humaidan P, Alsbjerg B. GnRHa trigger for final oocyte maturation: is HCG trigger history? Reprod Biomed Online 2014;29(3):274-280

66 Bermejo A, Cerrillo M, Ruiz-Alonso M, et al. Impact of final oocyte maturation using gonadotropin-releasing hormone agonist triggering and different luteal support protocols on endometrial gene expression. Fertil Steril 2014;101(1):138-146.e3

67 Toner JP. Vaginal delivery of progesterone in donor oocyte therapy. Hum Reprod 2000;15(Suppl 1):166-171

68 Berger B, Phillips J. A retrospective analysis of pregnancy outcomes in recipients of anonymously donated oocytes at a large ART center. Fertil Steril 2007;89:S11-S12
69 Berger B, Phillips J. A retrospective analysis of pregnancy outcomes in recipients of frozen/thawed embryos (FET) from donated oocytes at a large assisted reproductive technology (ART) center. Fertil Steril 2008;90:S459

70 Williams SC, Donahue J, Muasher SJ. Vaginal progesterone therapy during programmed cycles for frozen embryo transfer: an analysis of serum progesterone levels and pregnancy rates. Fertil Steril 2000;74(Suppl 1):S209

71 Kaser DJ, Ginsburg ES, Missmer SA, Correia KF, Racowsky C. Intramuscular progesterone versus $8 \%$ Crinone vaginal gel for luteal phase support for day 3 cryopreserved embryo transfer. Fertil Steril 2012;98(6):1464-1469

72 Shapiro DB, Pappadakis JA, Ellsworth NM, Hait HI, Nagy ZP. Progesterone replacement with vaginal gel versus i.m. injection: cycle and pregnancy outcomes in IVF patients receiving vitrified blastocysts. Hum Reprod 2014;29(8):1706-1711

73 Muasher S, Acosta AA, Garcia JE, Jones GS, Jones HW Jr. Luteal phase serum estradiol and progesterone in in vitro fertilization. Fertil Steril 1984;41(6):838-843

74 Sharara FI, McClamrock HD. Ratio of oestradiol concentration on the day of human chorionic gonadotrophin administration to mid-luteal oestradiol concentration is predictive of in-vitro fertilization outcome. Hum Reprod 1999;14(11): 2777-2782

75 Kolibianakis EM, Venetis CA, Papanikolaou EG, Diedrich K, Tarlatzis BC, Griesinger G. Estrogen addition to progesterone for luteal phase support in cycles stimulated with $\mathrm{GnRH}$ analogues and gonadotrophins for IVF: a systematic review and meta-analysis. Hum Reprod 2008;23(6):1346-1354

76 Jee BC, Suh CS, Kim SH, Kim YB, Moon SY. Effects of estradiol supplementation during the luteal phase of in vitro fertilization cycles: a meta-analysis. Fertil Steril 2010;93(2):428-436

77 Elgindy EA, El-Haieg DO, Mostafa MI, Shafiek M. Does luteal estradiol supplementation have a role in long agonist cycles? Fertil Steril 2010;93(7):2182-2188

78 Lin H, Li Y, Li L, et al. Oral oestradiol supplementation as luteal support in IVF/ICSI cycles: a prospective, randomized controlled study. Eur J Obstet Gynecol Reprod Biol 2013;167(2): $171-175$ 